ASE-14-0100

Research Report

\title{
Application of the One-Minute Preceptor Technique by Novice Teachers in the Gross Anatomy Laboratory
}

\author{
Lap Ki Chan ${ }^{1,2} *$, Jian Yang ${ }^{2}$, David M. Irby ${ }^{3,4}$ \\ ${ }^{1}$ Institute of Medical and Health Sciences Education, Li Ka Shing Faculty of Medicine, The \\ University of Hong Kong, Hong Kong SAR, China \\ ${ }^{2}$ Department of Anatomy, Li Ka Shing Faculty of Medicine, The University of Hong Kong, Hong \\ Kong SAR, China \\ ${ }^{3}$ Department of Medicine, Division of General Internal Medicine, University of California San \\ Francisco, San Francisco, California \\ ${ }^{4}$ Office of Research and Development in Medical Education, University of California San \\ Francisco, San Francisco, California
}

Running title: Application of One-Minute Preceptor Technique by Novice Teachers

*Correspondence to: Dr. Lap Ki Chan, Institute of Medical and Health Sciences Education, Department of Anatomy, Li Ka Shing Faculty of Medicine, The University of Hong Kong, 2/F, William MW Mong Block, Faculty of Medicine Building, 21 Sassoon Road, Pokfulam, Hong Kong SAR, China. E-mail: lapki@hku.hk 


\section{ABSTRACT}

The one-minute preceptor (OMP) was originally developed in the ambulatory care setting as a time-efficient teaching technique for learner-centered clinical training. There are also possible advantages of using the OMP in the gross anatomy laboratory. However, in a previous study it was found that providing training to experienced gross anatomy teachers in the use of the OMP did not result in improvement in students' perceptions of their learning, probably due to the fact that the experienced teachers had already developed their own pedagogical approaches. In the current study, we examined the effects of training novice teachers with about four years of gross anatomy teaching experience, in the use of the OMP in the gross anatomy laboratory, by surveying students to collect their views on their learning experiences, by observing the teachers' teaching behaviors before and after they were trained in OMP, and then by interviewing them. More students reported a better learning experience in the session after the teachers had been trained in the OMP than reported worse, in eight out of the nine items related to their learning experiences. The novice teachers were receptive to the OMP. After the OMP training, the novice teachers were observed to engage more in getting commitments from the students and in reinforcing what the students have done right, two of the five OMP microskills. They considered the OMP to be very useful for their development as anatomy teachers.

Keywords: gross anatomy education, undergraduate education, medical education, learning and teaching, faculty development, junior faculty, teaching skills, teaching basic sciences 


\section{INTRODUCTION}

Novice faculty members, especially in Anatomy, struggle to develop and hone their teaching skills. Faculty development, which seeks to improve faculty performance in teaching, research and administration (Ouellett, 2010), is commonly used to enhance faculty members’ teaching abilities (Rudland, 2005; Lee, 2010). However, teaching in gross anatomy laboratories has received little attention, even though it is a complex teaching environment that allows much faculty-student interactions (Drake, 1998). Apart from anatomy knowledge, students also learn nontraditional discipline-independent skills, such as communication skills, interpersonal skills and leadership skills (Pawlina et al., 2006; Pawlina and Drake, 2008). In the laboratory, teachers have to help in cadaveric dissection (if it is used) and the identification of anatomical structures, and facilitate discussions of functions, embryology and clinical relevance of the structures being dissected or observed. Frequently, teachers need to supervise several groups of students in a large gross anatomy laboratory and can thus spend only a limited time with each group. All of this demands a good blend of content knowledge, dissection skills, time management skills and group discussion facilitation skills. Teaching in the gross anatomy laboratory is difficult for novice teachers because teaching encounters are short, highly variable and complicated.

One potential strategy for advancing novice anatomy teachers' pedagogical skills in the anatomy laboratory is the one-minute preceptor model (Chan and Wiseman, 2011), which was originally designed for use by busy ambulatory care practitioners (Neher et al., 1992). The intent of the model, as applied in the ambulatory care setting, is to create minimal disruption to clinical care while allowing learners to acquire vital information in a learner-oriented fashion. The phrase “one-minute” does not imply a time restriction, but rather a highly efficient approach, which is 
particularly important when teaching time is limited (Conn et al., 2012). The OMP strategy enables preceptors to actively engage learners in problem-solving, foster learners’ responsibility for their own learning, customize the teaching activities at the learners' level of knowledge, and emphasize feedback and learners' reflection (Chan and Wiseman, 2011).

The OMP consists of five simple, discrete steps called microskills (Neher et al., 1992; Wall, 2008; Chan and Sharma, 2014), which can be implemented flexibly in different sequences and combinations to accommodate different teaching requirements:

1. Getting a commitment: stimulate students to tackle the proposed question using their existing knowledge, with a counter question such as "What do you think this is?"

2. Probing for supporting evidence: ask students for the reasons behind their response, before giving the answer. This step also provides a window into the students' knowledge and the reasoning processes so that teaching can be targeted to the learners' needs. Different types of questions can be used in this step to check the level of knowledge of the students (Lake et al., 2005).

3. Reinforcing what was done right: whether students give a correct or incorrect answer, the teacher can provide positive feedback on partially or completely correct responses, which forms an important part of any learning process (Lake, 2004; Lake and Ryan, 2005). The aim is to create a positive learning environment, encourage the students to solve problems by themselves, help them to build self-confidence, and reinforce the right behaviors.

4. Correcting mistakes and filling in omissions: clearly identify gaps and mistakes in the students' knowledge as well as the errors in reasoning, using objective, nonjudgmental language. 
5. Teaching a general rule or concept: based on the correct or erroneous responses, the teacher can direct their teaching to a key point that the students need to know. The aim is to enable students to apply what they have learned from a specific teaching encounter to other similar situations.

In prior research, residents who were trained to use the OMP produced better learning outcomes in their students, improved students' self-confidence in the use of their own knowledge to tackle other problems (Salerno et al., 2002), helped preceptors to focus more on disease-specific teaching (Irby et al., 2004), and enabled clinicians to ensure both educational sufficiency and effective patient care in ambulatory settings (Ferenchick et al., 1997). Furthermore, with the practical and effective framework provided by the OMP, preceptors are in a better position to structure interactions that are more conducive to learning. The OMP microskills are also easy to follow yet contain essential elements for promoting effective student-oriented learning; thus they should be especially beneficial for novice teachers.

The teaching environment of the gross anatomy laboratory is similar to that of the ambulatory care setting in that teacher-student encounters are also necessarily brief because one teacher oftentimes needs to supervise several groups of students in a large gross anatomy laboratory. Because the OMP is based on learning principles, it should be beneficial to learning in other settings. Chan and Wiseman (2011) thus proposed that the OMP could be adapted to gross anatomy teaching and would effectively prepare students for future clinical training. Chan and Sharma (2014) have since conducted studies involving the training of experienced gross anatomy teachers (with over 25 years of teaching experience) in the use of the OMP and evaluated the 
effects of the OMP on student perceived learning. Second-year medical students (mean age 20.1, standard deviation 1.7) from a five-year, undergraduate-entry curriculum were divided randomly into two groups to participate in the same gross anatomy practical session on different dates. Experienced teachers were trained in the OMP technique between the two sessions. The students were surveyed on their perceived learning experience at the end of each practical session. The results showed no improvement of students' learning perceptions after the teachers had been trained in the OMP. Experienced teachers had already developed their own teaching strategies, which incorporated elements similar to those in the OMP before their OMP training. They were better able to apply their own approaches with greater flexibility than they were to incorporate the five microskills of the OMP model. These experienced teachers commented that they would have benefited from being exposed to the OMP model when they were novice teachers.

The teaching and learning environment of the gross anatomy laboratory seems to present novice teachers with particular difficulties, as lamented by the experienced teachers in the study by Chan and Sharma (2014). As a logical follow-up of that study, this research focuses on novice gross anatomy teachers with less than five years of teaching experience, by evaluating students' perception of their learning experiences and by observing the teaching behaviors of these teachers, before and after they were trained in the OMP.

\section{METHODS}

The study was conducted on novice gross anatomy teachers and second year medical students at the Li Ka Shing Faculty of Medicine of The University of Hong Kong (HKU), with the approval of the Institutional Review Board of The University of Hong Kong/Hospital Authority Hong 
Kong West Cluster (HKU/HA HKW IRB). All participants voluntarily took part in the study. The average age of the students at the time of the study was 20.4, with a standard deviation of 1.2.

In 2012, all undergraduate programs funded by the University Grant Committee of Hong Kong, thus including the medical program at HKU, were extended by an additional year. Therefore the previous five-year MBBS program became a six-year program. The students involved in Chan and Sharma's study (2014) belonged to the five-year MBBS curriculum, while the students in this study belong to the six-year curriculum. However, the basic structure of the curriculum remained unchanged. It is an undergraduate-entry program with the majority of students entering the program immediately after they have finished their high school education. English is the sole language of instruction in the medical program (except in a Chinese language course and a small part of a clinical communication course), and the curriculum is problem-based and system-based. Horizontal integration is achieved through integrating basic sciences and clinical disciplines in the system blocks in the first three years of the curriculum, while vertical integration is realized by clinical exposure and problem-based learning using clinical cases in the early years and the revisiting of basic sciences in latter years of the curriculum. Gross anatomy is taught not as a distinct, independent discipline, but integrated into the various blocks, mostly in the first three years of the six-year curriculum. The learning of gross anatomy across all system blocks comprises 51 hours of lectures, 40.5 hours of practicals involving dissection, and 21.5 hours on prosected specimens and models. This study spanned two consecutive dissection sessions in the gastrointestinal block in the second semester of the second year. The two sessions were eight days apart and lasted for three hours each. The first session was on abdominal content while the second session was on the posterior abdominal wall. Dissection sessions are not mandatory and 
therefore not all students attended them. Because the novice teachers were trained in the use of OMP between the two dissection sessions, these two dissection sessions are called pre-OMP and post-OMP sessions.

In each of these dissection sessions, there were five gross anatomy teachers. Four of the five taught in both sessions and one of these four is an author of this paper (LKC), who was therefore excluded from the study. The remaining three teachers (coded as T1, T2 and T3) joined the Department of Anatomy four years prior, before which they did not have gross anatomy teaching experience. Their main teaching responsibilities included giving lectures, facilitating PBL tutorials and supervising dissection and demonstrations. During the dissection sessions, each teacher supervised four dissection groups, each with at most ten students working on the same cadaver. None of the teachers had been exposed to the OMP prior to the workshop offered in this study.

The three novice teachers went through a workshop on the OMP, organized and run by two of the three authors (LKC and JY), between the two dissection sessions, designed according to Merrill's four-part approach, consisting of presentation, examples, practice and feedback (Merrill, 1994). The workshop consisted of a 20 -minute talk on the principles of the OMP, a 20-minute group discussion and a 20-minute role-play session. The presentation focused on the principles, steps and applications of the OMP; how it has been used successfully in the ambulatory care setting (with examples); how it can be applied in the gross anatomy laboratory; and how the five microskills can be flexibly applied. At the end of the workshop, the three novice teachers were able to describe the five OMP microskills, analyze the theoretical advantages of the OMP, and 
demonstrate the application of the OMP in role plays. They were therefore considered to have achieved the intended learning outcomes of the workshop. There was no written assessment.

\section{Survey of student learning experiences}

In the pre-OMP and post-OMP dissection sessions, we explicitly instructed the students not to move to other tables, which they were encouraged to do in other dissection sessions. This ensured that students belonging to a dissection group supervised by T1, T2 or T3 were only taught by the assigned respective teacher.

In the pre-OMP session, students who were supervised by the three novice teachers were invited to complete a questionnaire that is the same as the one used in the OMP study by Chan and Sharma (2014). It contained nine statements on students' learning experience in the dissection session (see Table 1 for the statements) and these statements were constructed on the basis of the theoretical advantages of the OMP as expounded by Chan and Sharma (2014). In the post-OMP session, the students who were supervised by the three novice teachers and who had attended the pre-OMP session were invited to complete a questionnaire that contained the same nine statements as in the pre-OMP questionnaire. These students were also asked to compare the preOMP and the post-OMP sessions in regards to their learning experiences described in the nine statements. The students rated their degree of agreement with the statements on a five-point Likert scale. Wilcoxon signed-rank test was performed to compare the pre-OMP and post-OMP scores, with statistical significance set at $\mathrm{P}<0.05$, using SPSS Statistics version 19.

Students were informed on the information sheet accompanying the written consent that the 
project was about the OMP, but the students did not know in which session the OMP model was used.

\section{Observation of teaching behaviors}

During the complete period of both pre-OMP and post-OMP sessions (three hours each), each teacher was observed by a different observer. For each teacher-student encounter in the dissection sessions, the observer recorded any OMP steps that he or she could observe. The observers had been trained in another workshop on the OMP principles and on the recognition of the OMP steps but none of them had any training in gross anatomy. The observers were postgraduate students and research assistants, who were not in the Department of Anatomy and were not coworkers of the novice teachers. The research assistants did not know the novice teachers or the MBBS students before their first meeting in the pre-OMP session, and did not work for or with these teachers. At the end of each session, the three observers delivered their anonymized reports together to the authors, who therefore had no knowledge of which record belonged to which novice teacher. This was made known to the novice teachers so that they did not view such observation as assessment.

\section{Semistructured interviews of teachers}

Between one to two weeks after the post-OMP session, the three novice teachers were interviewed and asked about their experiences using the OMP. The interviews were transcribed and thematically analyzed using the qualitative data analysis software Nvivo 8.

\section{RESULTS}




\section{Survey of student learning experiences}

We distributed 104 questionnaires to students in the pre-OMP session and collected 74, resulting in a $71 \%$ return rate. In the post-OMP session, we distributed 75 questionnaires and collected 49 from students who attended both the pre-OMP and post-OMP sessions. The return rate was $65 \%$.

Table 1 summarizes the students' responses to the nine statements about learning experiences that are the same in both the pre-OMP and the post-OMP questionnaires. There were no significant differences (Wilcoxon signed-rank test, $\mathrm{P}<0.05$ ) between the ratings of the pre-OMP and post-OMP sessions, on all nine statements.

Table 2 summarizes the students' responses to nine additional statements in the post-OMP questionnaire that directly compared the learning experiences in the pre-OMP to the post-OMP sessions. For eight out of these nine statements, more students reported better learning experiences in the post-OMP than reported worse. The difference is particularly large for these four items: "The teaching interaction stimulated me to think more," "The teachers were able to understand my level of anatomy knowledge and teach me accordingly," "The teachers evaluated my knowledge of anatomical facts and my analytical skills" and "The teachers gave me positive feedback on things I did correctly.” For these eight statements, around $80 \%$ of the students perceived that the learning experience of the post-OMP session was equal to or better than that of the pre-OMP session. The only exception is the statement "The teachers offered suggestions for improvement," for which slightly more students reported a worse learning experience in the postOMP session and only $71 \%$ of students reported an equal or better learning experience in the post-OMP session. 
(Insert Table 1 and 2 here)

\section{Observations of teaching behaviors}

Table 3 summarizes the results of the observations of pre-OMP and post-OMP laboratory sessions. In the post-OMP dissection session, notably more microskills were used by the three novice teachers. Figure 1 shows the number of individual OMP steps per encounter in the preOMP and post-OMP sessions. In the post-OMP session, four of the five OMP steps had higher occurrence per encounter, with the only exception being "correct mistakes and fill in omissions." Out of all the OMP steps, the most often used is "get a commitment," for all three novice teachers in both pre-OMP and post-OMP sessions (but more often in the post-OMP session). There was no "probe for supporting evidence" or "teach a general rule" recorded with any of the three novice teachers in the pre-OMP dissection session.

(Insert Table 3 here)

An encounter with most of the OMP steps went like this. When a group of students asked one of the novice teachers where the spleen is after they have opened the abdomen of the cadaver, the teacher asked where the students think it is ( $\mathrm{C}$ - Get a commitment). The students then pointed at part of the stomach protruding out from the left costal margin. The teacher then invited the students to explain why they thought that was the spleen (E - Probe for supporting evidence). Students said because it is a structure with a smooth surface in the left upper quadrant. The teacher commented that the spleen is indeed a structure with smooth surface and lies in the left upper quadrant ( $\mathrm{R}$ - Reinforce what was done right). The teacher then proceeded to reach to the back of the stomach with his right hand and drag out the spleen. This was to correct the students' 
mistakes by showing that what the students identified was the stomach and where the spleen actually was ( $\mathrm{M}$ - Correct mistakes and fill in omissions). Finally, the teacher made a comment on structure identification in general ( $G$ - Teach a general rule): "The spleen is, in fact, quite a bit smaller than the stomach and lies quite posteriorly, close to the 9th to 11th ribs, between the fundus of the stomach and the diaphragm. So in the future, when you run into an identification difficulty, first identify something easy, which, in our case, should be the stomach, a large and empty viscera in the right upper quadrant. Based on what you learned about the spatial relationship between the stomach and the spleen, you should thus reach posteriorly into the space between stomach and the rib cage to look for the spleen”.

\section{Semistructured interviews of teachers}

All three teachers reported that they had no specific guidelines in their minds for interacting with students during anatomy laboratory sessions before the OMP workshop.

"No specific guidelines...I have been doing something similar.” (T1)

They all agreed that the OMP teaching style is useful for both teachers and students in gross anatomy laboratory sessions, except for students who are not willing to explore. They all strongly agreed that the OMP-style teaching stimulates the students to think more in their learning in the gross anatomy laboratory.

"Yes ... more interactive ... going through the process together with students... a problem trouble shooting process for me and students.” (T3)

"Not useful to some students who just want an easy way out and the answers right away" (T1)

“...some students like it, some others don't like it.” (T1)

The three teachers all agreed that using the OMP allows teachers to have better knowledge of the 
students' level of anatomy knowledge and to adjust their teaching accordingly. And they were consciously giving more feedback to the students when using the OMP.

"I believe that OMP helps a teacher understand the level of competence of the students...judging from their answers.” (T2)

"Yes...before OMP...a little feedback... now I realized that it’s not enough.” (T1)

Two of the three teachers reported that it was difficult to use OMP techniques for certain demanding questions that required the teachers to have extensive anatomical knowledge.

“As a novice teacher...I don't know [the answer to] some questions...may not be able to identify the structure or locate the structure.” (T1)

One teacher worried that the OMP-style teaching might affect the teacher's evaluation.

"The temptation to show off my knowledge ... worry that students think I am incompetent ... I may not appear as passionate as other teachers.” (T2)

But all three teachers expressed that they would keep applying the OMP-style teaching and improving their OMP skills in the future.

"Yes... will use more of the 5 steps of OMP... in different combination based on the situation.” (T1)

\section{DISCUSSION}

Student perceived learning experience

The survey of student perceived learning experiences in the pre-OMP and post-OMP sessions revealed no significant differences. This result is similar to that of other studies, which showed that the OMP training of teachers did not always result in improved learner perceptions (Salerno et al., 2002; Eckstrom et al., 2006). But when students were asked to directly compare their 
learning experiences before and after their teachers had been trained in OMP, most students reported better learning experiences in the post-OMP. Between $71 \%$ and $86 \%$ of the students indicated that their post-OMP experience was better than or equal to their pre-OMP experience.

These results are quite different from the findings following the training of very experienced gross anatomy teachers (Chan and Sharma, 2014), wherein a significant deterioration in the students' perception of two specific aspects of their learning occurred, namely "The teaching interaction stimulated me to think more” and“The teachers were able to understand my level of anatomy knowledge and teach me accordingly.” A likely explanation for the difference in results of the two studies, based on the teachers' interview and their gross anatomy teaching experience level, is that the experienced teachers had already developed their own teaching scripts before OMP training, while the novice teachers had not. The experienced teachers had already incorporated many elements of OMP into their approaches to interacting with students: being learner centered, adaptable to students’ learning needs, and providing feedback. Altering the teaching behaviors of experienced teachers by asking them to adopt new teaching routines, according to script theory (Tomkins, 1978), may have decreased their teaching performance until the new scripts could be equally automated. But for the novice teachers, whose scripts are only forming, the OMP training workshop provided thfem with a compact, easy-to-follow and educationally sound script and therefore was readily adopted. In the interviews with the novice teachers, the teachers commented on how useful the OMP technique was in structuring teacherstudent interactions and how easy it was to adopt and apply. Unlike what happened with the experienced teachers, the OMP training did not seem to lead to unnatural (as perceived by the teachers) student-teacher interactions, probably because there were no well-established scripts 
that would interfere with the OMP script.

Among the nine statements about students' learning experiences, there was only one statement for which more students indicated that it was worse in the post-OMP session than those who indicated that it was better: "The teachers offered suggestions for improvement.” OMP stimulates students to think and reflect on their prior knowledge. In that process, a structure identification question can lead to other related questions (on, say, function, development and clinical relevance) and a dialogue between the teacher and the students. To give sound advice or useful suggestions in those situations requires the teachers to have broader and deeper knowledge as well as skills in leading the discussion. That is why the teachers said in their interviews that they needed a stronger knowledge base in order to make the best of OMP to stimulate student learning. The lack of such knowledge and pedagogical skills in the novice teachers may be the reason for more students saying that the post-OMP was worse than the preOMP concerning the statement of "The teachers offered suggestions for improvement." Alternatively, students may have expected teachers to provide direct instruction rather than ask questions. Since the students were asked to make a commitment first, they may have perceived that answers were not forthcoming as frequently as before. Indeed, the observations of the teachers shows that these novice teachers provided very few teaching points as general rules or principles.

When the students were asked to compare their pre-OMP and post-OMP experience, there were some students who perceived that the post-OMP was worse (though still fewer than those who perceived that it was better, in eight out of nine statements). This finding may reflect the fact that 
the majority of students entered the medical program immediately after they finished their high school education, wherein didactic teaching remains the main pedagogy. Students needed to adapt to a learner-centered pedagogy like the OMP and some may still prefer the didactic pedagogy that they were used to. Some students may think memorization is more important than the higher levels of learning. However, the MBBS curriculum at HKU is a hybrid problem-based curriculum, with PBL starting in the first year of the program and occupying about $20 \%$ of the contact hours. Therefore by the time students reach their second year (i.e., when this study took place), they have been exposed to PBL for at least a year. PBL is also a more interactive way of learning through discussion, like those OMP encounters in the gross anatomy laboratory.

Other factors that may also affect students' perception of the OMP are their personality and selfconfidence (Chan and Sharma, 2014). Teachers commented that some students were shy and it was difficult to get these students to commit to an answer. These students may not prefer the OMP since it is stressful for them. When teachers do encounter shy students, they should probably first try to create a supportive learning climate by making clear that any answer is acceptable and that no answer is "too stupid" (Lake et al., 2005). The OMP steps of reinforcing what was done right and correcting mistakes and filling in omissions is a feedback-giving process aimed at establishing a positive environment, which is similar to the Pendleton model of feedback giving (Vickery and Lake, 2005). Teachers need to be aware of the different learning needs and styles of the learners under different circumstances (Lake, 2004). Therefore, we consider that the OMP is a technique that should be built on top of more basic skills of facilitating small group learning, which includes techniques for engaging students with different personalities and learning styles. 
How novice teachers used the OMP

Observation results demonstrated notable differences in the novice teachers' teaching behaviors between the pre-OMP session and post-OMP session (132 OMP steps in 89 encounters vs. 68 steps in 63 encounters). This result is consistent with a prior study (Furney et al. 2001) that found changes in resident teaching behaviors after OMP training, despite no significant change in the perceived overall teaching effectiveness ratings by students.

The observation results showed that the increase in the use of OMP steps were mainly on C (Get a commitment) and $\mathrm{R}$ (Reinforce what was done right). The total counts of $\mathrm{C}$ increased from 38 in the pre-OMP session to 86 in the post-OMP session, and R from 21 to 36 . There were no E (Probe for supporting evidence) or G (Teach a general rule) observed in the pre-OMP session, and only $3 \mathrm{E}$ and $2 \mathrm{G}$ in the post-OMP session. $\mathrm{M}$ (Correct mistakes and fill in omissions) actually decreased in the post-OMP session (9 in the pre-OMP and 5 in the post-OMP session).

Both the experienced teachers and the novice teachers commented that the most difficult microskill to apply was G, as there were not as many general rules in gross anatomy as in clinical settings, and teaching a general rule in the gross anatomy laboratory is challenging (Chan and Wiseman 2011). As reported by Furney et al. (2001), teaching a general rule was the microskill most difficult for teachers to develop even in the application of the OMP to clinical case presentations. Perhaps if the anatomy teachers (novice and experienced) had realized that the intent of teaching a general rule is to teach students what to do next time, this might not have been so difficult. 
Probing for supporting evidence (E) was also not observed frequently; there were none in the pre-OMP and only 3 in the post-OMP sessions. It appeared that the novice teachers oftentimes moved from getting a commitment (C) to reinforcing what is right (R), skipping the intermediate step of exploring for supporting evidence. Exploring for supporting evidence helps the teacher to target instruction to the student's level of need. Although in the gross anatomy laboratory, the questions usually focus on the identification of structures and are much more straightforward than in clinical medicine, there is still the need to explore students' understanding. In the example given in the Results section, it is useful to ask why the students thought the stomach was the spleen, in order to help them overcome their confusion.

The novice teachers in general were receptive of the OMP. They felt that the OMP technique encouraged students to try to solve a problem themselves before asking for help, encouraged the teachers to engage in a dialogue, go through the problem-solving process with students and provide more positive feedback. The OMP offered the teachers greater insight into student knowledge and reasoning. This result is similar to the improved self-evaluation after OMP training as reported by Kertis (2007). The teachers nevertheless also mentioned a few challenges in using the OMP. It was not always well received by students, especially shy students; it was more time-consuming; and it required the teachers to be more confident and have broader and deeper knowledge.

\section{Limitation of this study}


This study has a number of limitations, including a small sample size (three novice teachers), one university (The University of Hong Kong), and a limited intervention (a one hour faculty development workshop). Also this study did not directly examine the effects of the intervention on students' performance assessment. Summative assessment takes place at the end of the academic year and combines all disciplines since the MBBS curriculum at HKU is a systembased, problem-based curriculum and is not structured according to disciplines. Moreover, the part of the curriculum covered by the two dissection sessions is small compared to the whole year's content and will therefore be unlikely to result in a significant difference in the students' assessment scores. The novice teachers in this study also had limited time for the naturalization of the newly learned OMP skills (the post-OMP session was the first time the teachers applied the OMP). This may have contributed to the observation that only two of the five OMP steps had increased in the post-OMP session. Whether they will be retained and whether these teachers will use more of the other OMP microskills in the future still need to be studied. Nonetheless, this study provides useful information about the actual implementation of the five microskills as well as the perceptions of learning by students in a basic science setting. It also offers insights into the challenges associated with implementing and adapting new teaching scripts (Irby, 2014).

\section{CONCLUSION}

This study explored the effects of OMP training on novice teachers in a gross anatomy laboratory. Although the statistical result shows no improvement of students' perceptions on their learning, more students reported better learning experiences in the post-OMP than reported worse. The novice teachers appeared to be more receptive of the OMP than the experienced teachers and considered the OMP to be very useful for their development as anatomy teachers. After the OMP 
training, the novice teachers were observed to engage more in getting commitments from the students and in reinforcing what was done right, two of the five OMP microskills. Overall, the results indicate that the OMP has value in the development of novice teachers of anatomy. 


\section{ACKNOWLEDGMENTS}

The authors would like to thank Namkiu Chan, Henrietta Lai, Wai Kit Ming, Joyce Tsang, and Lily Wu for their valuable assistance in data collection, and serving as observers during the preOMP and post-OMP sessions. 


\section{NOTES ON CONTRIBUTORS}

LAP KI CHAN, M.B.B.S. (HK), F.H.K.A.M., F.H.K.C.O.S. (Orthopedics), F.R.C.S. (Edinburgh), Ph.D. (Duke), is an Assistant Dean (Pedagogy) at the Li Ka Shing Faculty of Medicine and an associate professor in the Institute of Medical and Health Sciences Education and the Department of Anatomy, The University of Hong Kong, Hong Kong SAR China. He has a background in orthopedics and physical anthropology and teaches gross anatomy to medical students.

JIAN YANG, Ph.D., is a lecturer in the Department of Anatomy, Li Ka Shing Faculty of Medicine, The University of Hong Kong, Hong Kong, SAR China. He teaches anatomy and histology to first-, second- and third-year medical students.

DAVID M. IRBY, PhD, is a professor in the Department of Medicine, School of Medicine, University of California San Francisco, San Francisco, California. He is the former vice dean for education, and now teaches and conducts research in medical education. 


\section{LITERATURE CITED}

Al-Umran K. 2004. Teaching tips - questioning. J Fam Community Med 11:73.

Chan LK, Wiseman J. 2011. Use of the one-minute preceptor as a teaching tool in the gross anatomy laboratory. Anat Sci Educ 4:235-238.

Chan LK, Sharma N. 2014. Effects of training experienced teachers in the use of the one-minute preceptor technique in the gross anatomy laboratory. Anat Sci Educ 7:124-129.

Conn JJ, Lake FR, McColl GJ, Bilszta JL, Woodward-Kron R. 2012. Clinical teaching and learning: from theory and research to application. Med J Aust 196:527.

Drake RL. 1998. Anatomy education in a changing medical curriculum. Anat Rec 253:28-31.

Drake RL, McBride JM, Lachman N, Pawlina W. 2009. Medical education in the anatomical sciences: The winds of change continue to blow. Anat Sci Educ 2:253-259.

Eckstrom E, Homer L, Bowen JL. 2006. Measuring outcomes of a one-minute preceptor faculty development workshop. J Gen Intern Med 21:410-414.

Ferenchick G, Simpson D, Blackman J, DaRosa D, Dunnington G. 1997. Strategies for efficient and effective teaching in the ambulatory care setting. Acad Med 72:277-280. 
Furney SL, Orsini AN, Orsetti KE, Stern DT, Gruppen LD, Irby DM. 2001. Teaching the one minute preceptor. J Gen Intern Med 16:620-624.

Irby DM. 2014 Excellence in clinical teaching: Knowledge transformation and development required. Med Educ 48:776-784.

Irby DM, Aagaard E, Teherani A. 2004. Teaching points identified by preceptors observing oneminute preceptor and traditional preceptor encounters. Acad Med 79:50-55.

Kertis M. 2007. The one-minute preceptor. J Nurses Staff Dev 23:238-242.

Lake FR. 2004. Teaching on the run tips: Doctors as teachers. Med J Aust 180:415-416.

Lake FR, Ryan G. 2005. Teaching on the run tips 8: Assessment and appraisal. Med J Aust 182:580-581.

Lake FR, Vickery AW, Ryan G. 2005. Teaching on the run tips 7: Effective use of questions. Med J Aust 182:126-127.

Lee VS. 2010. Program types and prototypes. In: Gillespie KJ, Robertson DL, (Editors). A Guide to Faculty Development. 2nd Ed. San Francisco, CA: Jossey-Bass. p 21-33.

Merrill MD. 1994. Instructional Design Theory. $1^{\text {st }}$ Ed. Englewood Cliffs, NJ: Educational 
Technology Publications. 465 p.

Neher JO, Gordon KC, Meyer B, Stevens N. 1992. A five step microskills model of clinical teaching. J Am Board Fam Pract 5:419-424.

Ouellett ML. 2010. Overview of faculty development. In: Gillespie KJ, Robertson DL, (Editors). A Guide to Faculty Development. 2nd Ed. San Francisco, CA: Jossey-Bass. p 3-20.

Pawlina W, Drake RL. 2008. Driving Effective Communication Through Anatomy. Anat Sci Educ 1:49.

Pawlina W, Hromanik MJ, Milanese TR, Dierkhising R, Viggiano TR, Carmichael SW. 2006. Leadership and professionalism curriculum in the gross anatomy course. Ann Acad Med Singapore 35:609-14.

Rudland JR. 2005. Learning in small groups. In: Dent JA, Harden RM (Editors). A Practical Guide for Medical Teachers. 2nd Ed. Edinburgh: Elsevier Churchill Livingstone. p 57-65.

Salerno SM, O’Malley PG, Pangaro LN, Wheeler GA, Moores LK, Jackson JL. 2002. Faculty development seminars based on the one-minute preceptor improve feedback in the ambulatory setting. J Gen Intern Med 17:779-787.

Tomkins SS. 1978. Script theory: Differential magnification of affects. Nebr Symp Motiv Paper 
26:201-236.

Vickery AW, Lake FR. 2005. Teaching on the run tips 10: Giving feedback. Med J Aust 183:267268.

Wall D. 2008. The straight facts no nonsense teacher. In: Mohanna K, Chambers R, Wall D (Editors). Your Teaching Style: A Practical Guide to Understanding, Developing and Improving. 1st Ed. Abingdon Oxon, UK: Radcliffe Publishing Ltd. p 53-60. 


\section{FIGURE LEGENDS}

Figure 1. Graph shows the number of individual OMP steps per encounter in the pre-OMP and post-OMP sessions. Data from the three novice teachers were pooled; C, Get a commitment; E, Probe for supporting evidence; $\mathbf{R}$, Reinforce what was done right; $\mathbf{M}$, Correct mistakes and fill in omissions; G, Teach a general rule.

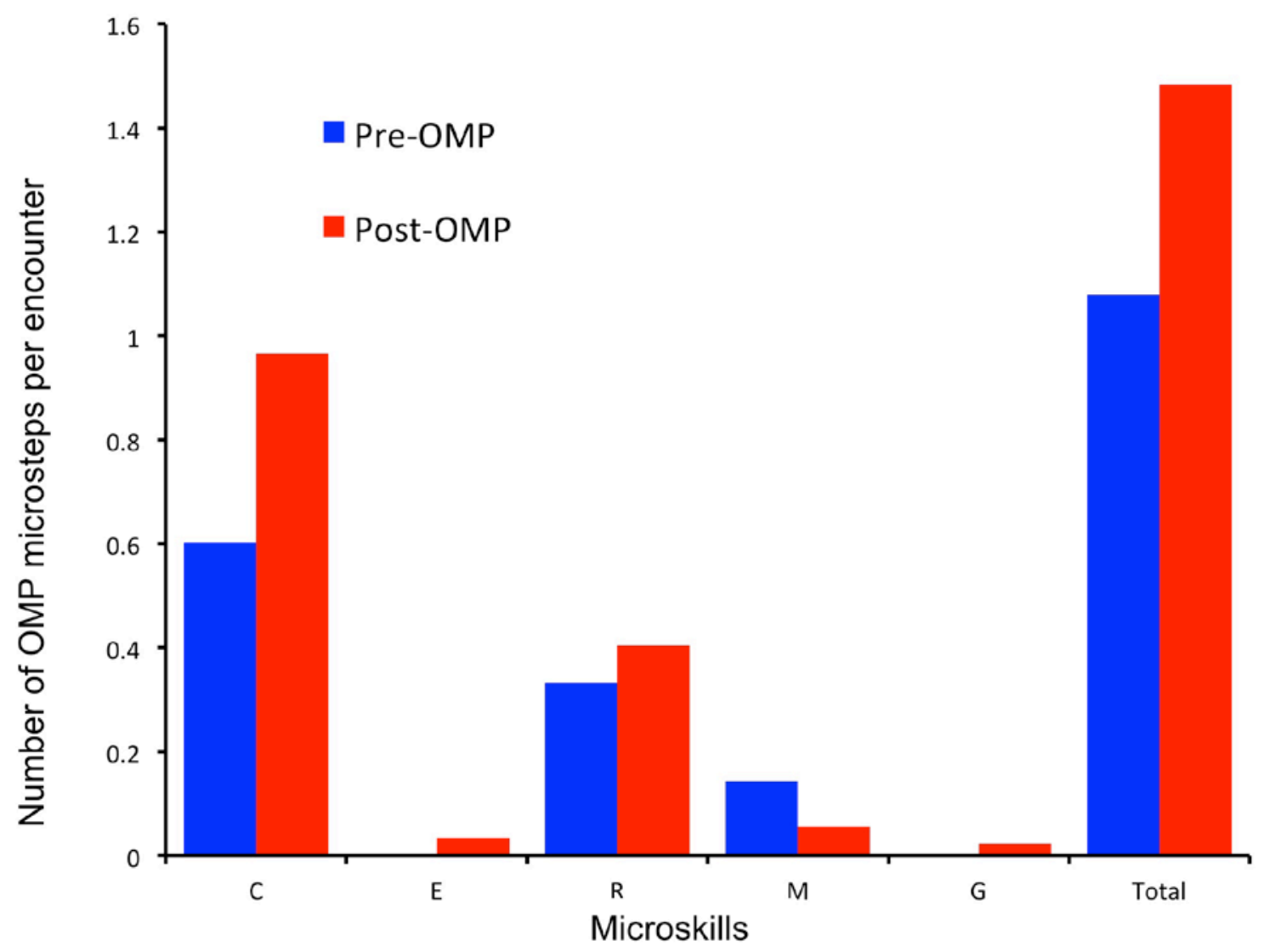

